\title{
Prediction of solar cycle 24 based on the Gnevyshev-Ohl-Kopecky rule and the three-cycle periodicity scheme
}

\author{
R. P. Kane \\ Instituto Nacional de Pesquisas Espacias, INPE C. P. 515 12201-970 São Jose dos Campos, SP, Brazil \\ Received: 12 June 2008 - Revised: 6 August 2008 - Accepted: 27 August 2008 - Published: 21 October 2008
}

\begin{abstract}
An examination of the maximum yearly values of the conventional sunspot number $R z$ of all cycles revealed fluctuations of various intervals in the high periodicity region (exceeding 11 years), namely 2 cycles (Hale, 22 years), 3 cycles (TRC, three-cycle) and longer intervals. The 2-cycle spacings had the smallest amplitudes. According to the G-O (Gnevyshev-Ohl) rule (Gnevyshev and Ohl, 1948), the evennumbered series of the maxima of annual mean Wolf sunspot numbers $R z$ are followed by higher amplitude odd-numbered series. Kopecky (1950) generalized this relation to annual mean Wolf numbers corresponding to equivalent phases of the adjacent even-odd 11-year cycles. Therefore, we would call it the G-O-K rule. For the data of 28 cycles (cycle -4 to cycle 23$)$, it was found that four pairs $(\sim 29 \%)$ from the fourteen even-odd pairs showed failure of the G-O-K rule. In the remaining ten pairs, the magnitudes of the odd cycles were well-correlated with the magnitudes of the preceding even cycles, but it was impossible to tell whether it would be a normal pair following the G-O-K rule or a possible case of failure. A much stronger sequence was the three-cycle sequence (TRC, low, high, higher). The 2-cycle oscillations were embedded into the TRC until the G-O-K rule failures occurred as in cycle 23. The patterns of cycle 17 (low), 18 (high), 19 (higher); 20 (low), 21 (high), 22 (higher) were noticed and used by Ahluwalia $(1995,1998)$ to predict a low value for cycle 23, which was accurate. However, in the earlier data, the preceding sequence $(14,15,16)$ was rather uncertain, and before that for seven cycles (cycles 8-14), there were no TRC sequences at all. During the twelve cycles -4 to 7 , there were only three isolated TRC sequences (one doubtful).

In view of this chequered history of TRC, it is doubtful whether the present TRC pattern (cycles 17-23) would persist in the near future. Spectral analysis showed that in the first half (cycles -4 to 9), larger periodicities (reminiscent of
\end{abstract}

Correspondence to: R. P. Kane

(kane@dge.inpe.br) the Gleissberg cycle of $\sim 80$ years) prevailed. but in the latter half, periodicities were different (3-year cycle was predominant) and the matching was not good. In particular, the points for the recent cycles 21,22 seemed to deviate considerably from the constructed series, thus introducing unreliability in predictions for the future by using extrapolation of periodicities.

Keywords. Solar physics, astrophysics, and astronomy (Corona and transition region; Flares and mass ejections; Photosphere and chromosphere)

\section{Introduction}

The predictions of the strength of a solar cycle are needed for many purposes, (satellite drag, operation of power grids on Earth and satellite communication systems etc.). Predictions are made using different methods. Many are based on sound physical principles, some are based on statistical analyses (including extrapolation of periodicities) and in some, association of successive cycles is explored (notably, evenodd cycles). Among these, one is based on what is known as the G-O (Gnevyshev-Ohl) rule (Gnevyshev and Ohl, 1948), which states that the even-numbered International Sunspot Number series 11-year cycles have been followed by higher amplitude odd-numbered series (Komitov and Bonev, 2001). The G-O rule was based on a statistical relationship between the annual mean Wolf sunspot numbers summed up over adjacent even- and following odd-numbered 11-year cycles, respectively. Kopecky (1950) generalized this relation to annual mean Wolf numbers corresponding to equivalent phases of the adjacent even-odd 11-year cycles. The physical assumption of the G-O rule (henceforth termed as the G-O-K rule) is that the fundamental cycle of the Sun is the 22-year magnetic (Hale) cycle, which consists of two 11-year oscillations and begins with an even-numbered one (Obridko,

Published by Copernicus Publications on behalf of the European Geosciences Union. 
1995). Javaraiah (2003) and later Javaraiah, Bertello and Ulrich (2005) found that the solar equatorial rotation rate during the odd-numbered sunspot cycles was well correlated with the equatorial rotation rate of the preceding even-numbered sunspot cycles, indicating that a 22 -year cycle in the equatorial rotation rate begins in an even-numbered cycle and ends in the following odd-numbered cycle. On the other hand, the latitudinal gradient of the solar rotation during the evennumbered cycles was found to be well correlated with that of the preceding odd-numbered cycles, indicating that a 22-year cycle in the latitudinal gradient begins in an odd-numbered cycle and ends in the following even-numbered cycle. Thus, the the beginning phase of a 22-year cycle in the latitudinal gradient is different by about $180^{\circ}$ relative to the beginning of a 22-year magnetic cycle. However, Javaraiah (2005) pointed out that it is not always possible to make a prediction for the odd cycle, because occasionally the G-O-K rule is violated. In a later paper, Javaraiah (2007) indicated that the values of sunspot areas at certain solar latitude belts are wellcorrelated with sunspot maxima of the next cyle. However, this does not involve the G-O-K effect as such, as a distinction is not made between even-odd and odd-even cycles.

Earlier, Komitov and Bonev (2001) had made a detailed analysis which revealed some specific discrepancies. For sunspot number data for 1749-1986 (end of cycle 21), they found that the G-O-K rule was valid for nine Hale cycles (even-odd pairs) but was violated twice, for 11-year cycles 45 and 8-9. Since by 2001, it was obvious that cycle 23 would be a low one (it turned out to have a maximum yearly value 119.5 ), the pair of even-odd cycles $22-23$ would be violating the G-O-K rule, as the maximum yearly value for cycle 22 was 157.8 . They attempted to examine the conditions for such violations by using a much longer data series, namely the Schove series (Schove, 1955, 1983), a set of reconstructed 11-year cycle characteristics providing the estimates for the years of minima and maxima and for the amplitudes of the 11-year cycles since BC 642. They concluded that the violations (an even cycle followed by a weaker odd cycle) occurred mostly when the even cycles were very powerful (maximum value exceeding 125 and decay times 6-year or more), however, with the exception of the even-odd pair 18 (even, 151.5)-19 (odd, 189.9). Meanwhile, Ahluwalia et al. (1996) and Ahluwalia (1998) had discovered a three-cycle quasi-periodicity in the magnetized solar wind from the polar coronal holes. They developed a forecast procedure and predicted that cycle 23 would be moderate, with a maximum of $132 \pm \sim 30$ in early 2000 . In spite of the strong criticisms from some solar astronomers, e.g. Wilson and Hathaway (1999) who were predicting a very strong cycle $23(\sim 200$, Wilson, 1998, and other references in Obridko; Oraevsky and Allen, 1994), the Ahluwalia et al. (1996) and Ahluwalia (1998) prediction came true (observed value $\sim 120$ ). Later, Ahluwalia (2003) reported the use of geomagnetic $a a$ index data for computing the rise time of a solar cycle and confirmed the validity of his prediction for cycle 23 . Thus, if a three-cycle periodicity exists, it will certainly complicate the operation of the G-O-K rule.

Both the even-odd effect and the three-cycle periodicity have been criticized by many co-workers, the even-odd effect by Joselyn et al. (1997), Komitov (1997), Komitov and Bonev (2001), Duhau (2003), Hathaway and Wilson, (2004), Komitov (2007), and probably many others. For the 3cycle periodicity suggested by Ahluwalia (1998), Wilson and Hathaway (1999) claimed that there were errors in the data used and on further examination of the longer sunspot record, it did not support the existence of the 3-cycle periodicity. Kane (2001a) came to a similar conclusion. Hathaway and Wilson (2004) also discounted the 3-cycle periodicity.

In the present paper, we re-examine the sunspot data and the operation of the G-O-K rule and other periodicities, and attempt a prediction for cycle 24 based on the observed value of cycle 23 and using the average patterns of the even-odd cycle effects and the 3-cycle periodicity scheme. However, we also emphasize that while such predictions can be made in principle, the correlations are small, standard errors are large, and the predictions are unreliable.

\section{Data}

The data used are for the conventional sunspot numbers $R z$. The sunspot values have been available since the last 300 years (since about 1700; Waldmeier, 1961), but the quality of the data is considered "poor" during 1700-1748, "questionable" during 1749-1817, "good" during 1818-1847, and "reliable" from 1848 onwards (McKinnon, 1987). A sunspot cycle ( $\sim 11$ years) is defined as one sunspot minimum to the next. (The interval $1755-1765$ is designated as Cycle 1). The sources and records of older sunspot data were examined critically by Hoyt and Schatten (1998a, b), who produced a new series of Group sunspot number $R_{G}$, which we propose to use. The $R_{G}$ data differ from the $R z$ data before 1882. The differences would have implications for the various published results obtained by using $R z$. Hoyt and Schatten (1997) had earlier re-examined the topics related to climate. Kane (2002) examined other topics and found that all results for the recent decades (1850 onwards) remained unaltered. The correlations between $R_{G}$ and $R z$ for successive 50year intervals were: +0.68 for $1700-1750 ;+0.88$ for $1751-$ $1800 ;+0.97$ for $1801-1850 ;+0.97$ for $1851-1900 ;+0.99$ for 1901-1950 and +0.99 for 1951-1990. (Ken Schatten informs us in private communication that, concerning Group numbers $R_{G}$, the funding for this project was not continued; so he and Doug Hoyt have not updated the group numbers beyond 1995). Figure 1 displays the annual values of $R z$ (full lines) and $R_{G}$ (crosses) for (a) $1700-1800$, (b) $1800-1900$, and (c) 1900-1995. The intervals (in years) refer to $R z$. As can be seen, the $R z$ values are larger than the $R_{G}$ values, much more so in the earlier years indicating that $R z$ is overestimated, as already mentioned by Hoyt and Schatten (1997, 1998a, b). 
Table 1. Maximum yearly values of $R z$ and $R_{G}$ for cycles -4 to 23 , their years of occurrence, and the deviation (\%) of $R_{G}$ values from the $R z$ values (last column).

\begin{tabular}{|c|c|c|c|c|c|c|c|}
\hline \multirow[t]{2}{*}{ Cycle } & \multicolumn{2}{|c|}{ Year of maximum } & \multicolumn{2}{|c|}{ Spacing (years) } & \multicolumn{2}{|c|}{ Maximum values } & \multirow{2}{*}{$\begin{array}{c}\text { Dev. \% } \\
\left(R z-R_{G}\right) / R z\end{array}$} \\
\hline & $R z$ & $R_{G}$ & $R z$ & $R_{G}$ & $R z$ & $R_{G}$ & \\
\hline-4 & 1705 & 1705 & & & 58 & 6 & 89.7 \\
\hline-3 & 1717 & 1719 & 12 & 14 & 63 & 34 & 46.0 \\
\hline-2 & 1727 & 1730 & 10 & 11 & 122 & 85 & 30.3 \\
\hline-1 & 1738 & 1739 & 11 & 9 & 111 & 56 & 49.5 \\
\hline 0 & 1750 & 1749 & 12 & 10 & 83 & 65 & 21.7 \\
\hline 1 & 1761 & 1761 & 11 & 12 & 86 & 74 & 14.0 \\
\hline 2 & 1769 & 1769 & 8 & 8 & 106 & 102 & 3.8 \\
\hline 3 & 1778 & 1779 & 9 & 10 & 154 & 80 & 48.1 \\
\hline 4 & 1787 & 1787 & 9 & 8 & 132 & 86 & 34.8 \\
\hline 5 & 1804 & 1801 & 17 & 14 & 48 & 50 & -4.2 \\
\hline 6 & 1816 & 1816 & 12 & 15 & 46 & 31 & 32.6 \\
\hline 7 & 1830 & 1830 & 14 & 14 & 71 & 64 & 9.9 \\
\hline 8 & 1837 & 1837 & 7 & 7 & 138 & 110 & 20.3 \\
\hline 9 & 1848 & 1848 & 11 & 11 & 125 & 86 & 31.2 \\
\hline 10 & 1860 & 1860 & 12 & 12 & 96 & 86 & 10.4 \\
\hline 11 & 1870 & 1870 & 10 & 10 & 139 & 96 & 30.9 \\
\hline 12 & 1883 & 1884 & 13 & 14 & 64 & 62 & 3.1 \\
\hline 13 & 1893 & 1894 & 10 & 10 & 85 & 88 & -3.5 \\
\hline 14 & 1905 & 1907 & 12 & 13 & 64 & 61 & 4.7 \\
\hline 15 & 1917 & 1917 & 12 & 10 & 104 & 110 & -5.8 \\
\hline 16 & 1928 & 1928 & 11 & 11 & 78 & 82 & -5.1 \\
\hline 17 & 1937 & 1937 & 9 & 9 & 114 & 121 & -6.1 \\
\hline 18 & 1947 & 1947 & 10 & 10 & 152 & 145 & 4.6 \\
\hline 19 & 1957 & 1958 & 10 & 11 & 190 & 175 & 7.9 \\
\hline 20 & 1968 & 1970 & 11 & 12 & 106 & 109 & -2.8 \\
\hline 21 & 1979 & 1979 & 11 & 9 & 155 & 155 & 0.0 \\
\hline 22 & 1989 & 1990 & 10 & 11 & 158 & 145 & 8.2 \\
\hline 23 & 2000 & & & & 120 & & \\
\hline
\end{tabular}

\section{Comparison of $R z$ and $R_{G}$ data}

Table 1 gives the maximum yearly values of $R z$ and $R_{G}$ for cycles -4 to 23 , their years of occurrence, and the deviation (\%) of $R_{G}$ values from the $R z$ values (last column). As can be seen, the deviations are considerable in the earlier data, and only from $\sim 1750$ onwards, the matching is reasonally good.

\section{The G-O-K rule results}

Table 2 illustrates the G-O-K rule. In the left half, successive even(e)-odd(o) cycles (columns 1 and 3) and their $R z(\mathrm{e})$ and $R z(\mathrm{o})$ values (columns 2 and 4 ) are given and the percentage excess of the value of the odd cycle over the value of the preceding even cycle $[(R z(\mathrm{o})-R z(\mathrm{e})) / R z(\mathrm{e})]$ is indicated (fifth column). (In the right half, similar values are given for $\left.R_{G}\right)$. As can be seen in the fifth column, many values are positive, indicating that the odd cycle values are larger than the even cycle values, conforming with the G-O-K rule.
However, values for even-odd pairs $(-2)-(-1), 4-5,8-9$ and 22-23 are negative, indicating a failure of the G-O-K rule (in agreement with Komitov and Bonev, 2001). From the fourteen pairs, ten support G-O-K rule while four do not support. Thus, the failure rate is $\sim 29 \%$, even qualitatively. Regarding quantities, the values in the fifth column have a large range, -64 to $+63 \%$, and the mean value is $19 \%$ with a standard deviation of $30 \%$. If the negative values are ignored, the range is 4 to $63 \%$ and the mean value is $37 \%$ with a standard deviation of $16 \%$. The results for $R_{G}$ in the right half of Table 2 are similar to those for $R z$. (For $R_{G}$, the values for cycle -4 are very small (only 6 ) and the percentage ratio with cyle -3 was abnormally large and these are omitted from further analysis).

Figure 2a shows the plots of $R z(\max )$ of even cycles (abscissa) versus $R z(\max )$ of odd cycles (ordinate) (values in the left half of Table 2 ). The full line is a $45^{\circ}$ line, implying equal values for even and odd cycles. Points below this line indicate failure of the G-O rule and are shown as open circles (four points, for even cycles, $-2,4,8,22$, same as reported 
Table 2. The G-O effect for even-odd pairs. In the left half, successive even(e)-odd(o) cycles (columns 1 and 3 ) and their $R z(\mathrm{e})$ and $R z(\mathrm{o})$ values (columns 2 and 4 ) are given and the percentage excess of the value of the odd cycle over the value of the preceding even cycle $[(R z(\mathrm{o})-R z(\mathrm{e})) / R z(\mathrm{e})]$ is indicated (fifth column). In the right half, similar values are given for $R_{G}$.

\begin{tabular}{|c|c|c|c|c|c|c|c|c|c|}
\hline $\begin{array}{l}\text { Col.1 } \\
\text { Even } \\
\text { cycle }\end{array}$ & $R z(\mathrm{e})$ & $\begin{array}{c}3 \\
\text { Odd } \\
\text { cycle }\end{array}$ & $R z(\mathrm{o})$ & $\begin{array}{c}5 \\
{[(R z(\mathrm{o})-R z(\mathrm{e})] / R z(\mathrm{e})} \\
\text { Percentage }\end{array}$ & $\begin{array}{c}6 \\
\text { Even } \\
\text { cycle }\end{array}$ & $R_{G}(\mathrm{e})$ & $\begin{array}{c}8 \\
\text { Odd } \\
\text { cycle }\end{array}$ & $\begin{array}{c}9 \\
R_{G}(\mathrm{o})\end{array}$ & $\begin{array}{c}10 \\
{\left[R_{G}(\mathrm{o})-R_{G}(\mathrm{e})\right] / R_{G}(\mathrm{e})} \\
\text { Percentage }\end{array}$ \\
\hline-4 & 58 & -3 & 63 & 8.6 & -4 & 6 & -3 & 34 & 467.7 \\
\hline-2 & 122 & -1 & 111 & -9.0 & -2 & 85 & -1 & 56 & -34.1 \\
\hline 0 & 83 & 1 & 86 & 3.6 & 0 & 65 & 1 & 74 & 13.8 \\
\hline 2 & 106 & 3 & 154 & 45.3 & 2 & 102 & 3 & 80 & -21.6 \\
\hline 4 & 132 & 5 & 48 & -63.6 & 4 & 86 & 5 & 50 & -41.9 \\
\hline 6 & 46 & 7 & 71 & 54.3 & 6 & 31 & 7 & 64 & 106.5 \\
\hline 8 & 138 & 9 & 125 & -9.4 & 8 & 110 & 9 & 86 & -21.8 \\
\hline 10 & 96 & 11 & 139 & 44.8 & 10 & 86 & 11 & 96 & 11.6 \\
\hline 12 & 64 & 13 & 85 & 32.8 & 12 & 62 & 13 & 88 & 41.9 \\
\hline 14 & 64 & 15 & 104 & 62.5 & 14 & 61 & 15 & 110 & 80.3 \\
\hline 16 & 78 & 17 & 114 & 46.2 & 16 & 82 & 17 & 121 & 47.6 \\
\hline 18 & 152 & 19 & 190 & 25.0 & 18 & 145 & 19 & 175 & 20.7 \\
\hline 20 & 106 & 21 & 155 & 46.2 & 20 & 109 & 21 & 156 & 43.1 \\
\hline \multirow{3}{*}{22} & 158 & 23 & 120 & -24.1 & 22 & 149 & 23 & & \\
\hline & & & Mean & 18.8 & & & & Mean & 20.5 \\
\hline & & & Std.dev. & 29.5 & & & & Std. Dev. & 36.2 \\
\hline
\end{tabular}

by Komitov and Bonev, 2001). Points above the line (shown as dots) are when G-O rule proved correct. Using all the pairs upto the even-odd pair cycles 20-21 (circles as well as dots), the correlation was only $+0.53 \pm 0.18$ (significant at a $2 \sigma$ level but low, due to the open circles).The regression equation (shown as the dashed line A in Fig. 2a) was:

$R z(\max )($ odd $)=(49.2 \pm 32.0)+(0.65 \pm 0.31) R z(\max )($ even $)$

Using the value $R z(\max )($ even$)=158$ for even cycle 22 in the right side of Eq. (1), the predicted $R z$ (max)(odd) for cycle 23 was $152 \pm 59$. The observed value of 120 was certainly in this range, but considering that the correlation was only +0.53 and the standard error of the predicted $R z(\max )$ (odd) was very large $( \pm 59)$, this scheme is unsatisfactory, because of the use of the points when the G-O-K rule failed. If the failure points (circles) were excluded from the analysis (arbitrarily, no justification), the correlation for only the dots was very high $(+0.94 \pm 0.03)$ and the regression equation (shown as the dashed line B in Fig. 2a) was:

$R z(\max )($ odd $)=(8.0 \pm 14.9)+(1.27 \pm 0.17) R z(\max )($ even $)(2)$

Using the value $R z(\max )($ even $)=158$ for even cycle 22 in the right side of Eq. (2), the predicted $R z(\max )(\mathrm{odd})$ for cycle 23 was $222 \pm 31$. The observed value of 120 was certainly much below this prediction and caused a lot of embarrassment to the co-workers believing in the G-O-K rule. Thus, this methodology is grossly unreliable.

Figure $2 \mathrm{~b}$ shows similar plots for $R_{G}$ (values in the right half of Table 2, omitting the pair cycle (-4)-(3)). Here, there are four circles but only three (cycles $-2,4,8$ ) are common to the $R z$ plot of Fig. 2a. In the $R_{G}$ plot, there is an additional circle for the odd-even pair cycles 2-3 (value 102/80, a failure of the G-O-K rule), while this pair was 106/154 in the $R z$ plot (a success of G-O-K rule). Since cycle 2 was in the early part of the data when $R z$ and $R_{G}$ differed considerably, we are not sure which values are correct. However, the point for the even-odd pair cycle 22-23, marked as a circle in Fig. 2a, is absent in Fig. 2b, because Hoyt and Schatten (private communication) could not calculate the $R_{G}$ values for 2000 (no data) when $R z$ (max) of cycle 23 occurred.

In Fig. 2b, using all the pairs upto the even-odd pair cycles 20-21 (circles as well as dots), the correlation was only $+0.61 \pm 0.16$ (significant at a $2 \sigma$ level but low, due to the open circles). The regression equation (shown as the dashed line $\mathrm{A}$ in Fig. 2b) was:

$$
R_{G}(\max )(\text { odd })=(28.6 \pm 29.6)+(0.79 \pm 0.33) R_{G}(\max )(\text { even })(3)
$$

Using the value $R_{G}(\max )$ (even)=149 for even cycle 22 (value in Table 2, right half) in the right side of Eq. (3), the predicted $R_{G}(\max )$ (odd) for cycle 23 was $146 \pm 58$. (Hoyt and Schatten have not calculated the $R_{G}(\max )$ value for cycle 23 , but it should be almost the same as for $R z(\max ))$. The observed value $R z(\max )=120$. was certainly in this range, but considering that the correlation was only +0.61 and the standard error of the predicted $R z(\max )($ odd $)$ was very large $( \pm 58)$, this scheme is unsatisfactory, because of the use of the points when the G-O-K rule failed. If the failure points (circles) were excluded from the analysis (arbitrarily, no justification), 
the correlation for the dots only was very high $(+0.92 \pm 0.03)$ and the regression equation (shown as the dashed line $\mathrm{B}$ in Fig. 2b) was:

$R_{G}(\max )($ odd $)=(27.8 \pm 15.3)+(1.03 \pm 0.18) R_{G}(\max )($ even $)(4)$

Using the value $R_{G}(\max )(\mathrm{even})=149$ for even cycle 22 in the right side of Eq. (4), the predicted $R_{G}$ (max)(odd) for cycle 23 was $181 \pm 31$. The observed value of 120 was certainly well below this prediction, again indicating that this methodology is unreliable.

Komitov and Bonev (2001) mention that the failures (even cycle followed by a weaker odd cycle) occur when the even cycles are very strong $(R z(\max )$ values exceeding 125$)$. This seems to be generally true for the four failures in Fig. 2a, namely even cycles -2 ( $R z(\max ) 122), 4$ (132), 8 (138) and 23 (158). However, there is also an exception, namely, cycle 18 (152) which was followed by a stronger cycle 19 (190) and conformed to the G-O-K rule. If the $28 R z(\max )$ values for cycles -4 to 23 , as given in Table 1, are subjected to autocorrelation with lags, a lag of one cycle (value of one cycle correlated with the value of the next cycle) results in a correlation of only $+0.37 \pm 0.20$, indicating that sunspot numbers of successive cycles are poorly correlated, and a significant prediction of the next cycle from values of the present cycle is not possible. Incidentally, better correlations are obtained if sunspot numbers at different solar latitudes (not whole disk but specific narrow solar latitude belts) are correlated with $R z$ (max) of the next cycle and thus, a prediction potential exists (Kane and Trivedi, 1980; Javaraiah, 2007; Kane, 2007a).

\section{Prediction for cycle 24 based on the G-O-K rule}

Equations (1-4) could be used (if at all) for estimating $R_{G}(\max )$ (odd) when $R_{G}(\max )($ even) values are available. Hence, the analysis ended with cycle 22 . However, since $R_{G}$ (max)(odd) for cycle 23 are available, these could be used for estimating the amplitude of cycle 24 if a reverse regression equation is available, namely, $R_{G}(\max )($ even) on the left side and $R_{G}(\max )(\mathrm{odd})$ on the right side. Such a regression equation can be obtained by using values in Table 3 . The correlation was insignificant, $+0.30 \pm 0.23$ and the regression equation was:

$R z(\max )($ even $)=(74.6 \pm 29.4)+(0.26 \pm 0.25) R z(\max )($ odd $)$.

Using the value $R_{G}$ (max)(odd)=120 for odd cycle 23 in the right side of Eq. (4), the predicted $R_{G}(\max )($ even) for cycle 24 is $106 \pm 42$. The large error is unsatisfactory.

Another way of obtaining this estimate was calculating the difference $R z(\mathrm{e})-R z(\mathrm{o})$ of Table 3 and calculate its mean and standard error. It was $-8 \pm 36$. Adding this to the value 120 for cycle 23 , the prediction for cycle 24 would be $112 \pm 36$, roughly the same as $106 \pm 42$. It may be noted that the standard errors are large because of the low correlation $+0.30 \pm 0.23$, and hence the prediction is in a larger range $\sim 60-150$, which is not at all satisfactory.

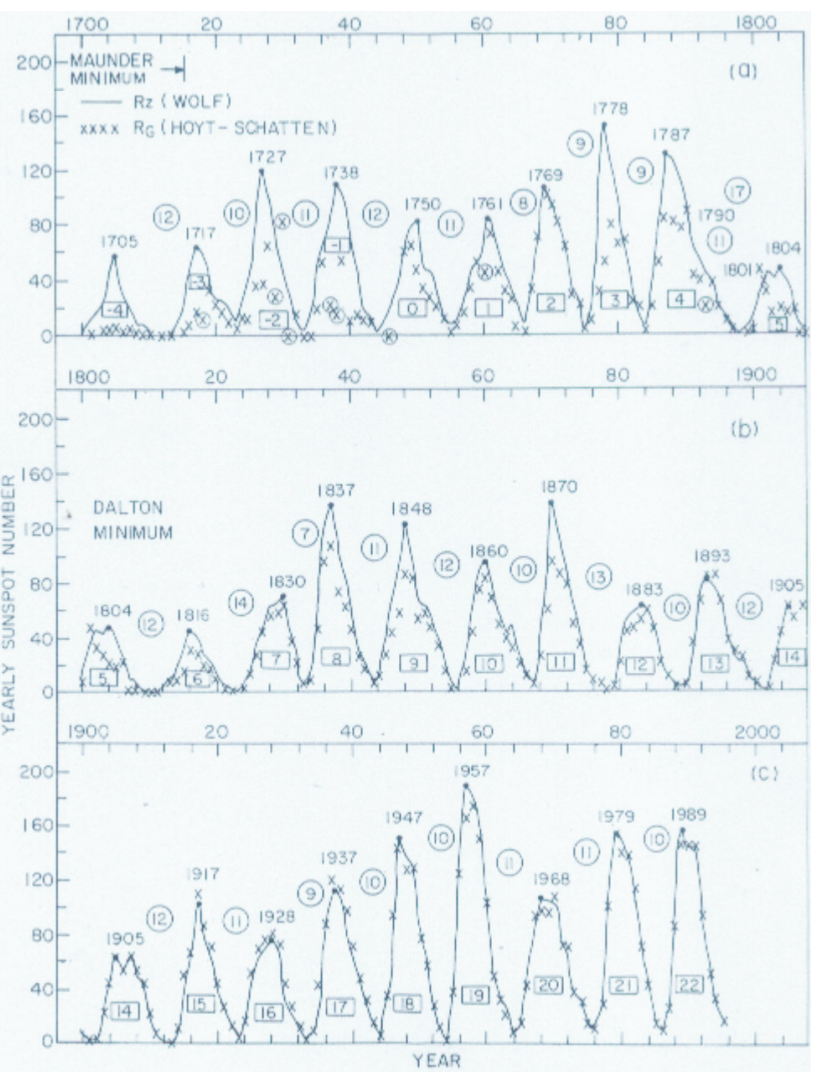

Fig. 1. Plots of annual values of $R z$ (full lines) and $R_{G}$ (crosses) for (a) 1700-1800. (b) 1800-1900, (c) 1900-1995, cycle numbers in rectangles, cycle spacings (in years, for $R z$ ) in circles.

\section{Three-cycle periodicity (TRC)}

The failure of the G-O-K rule could be partly due to interference by a three-cycle periodicity. Such a periodicity has been detected earlier in sunspot number series. Kane (1977) reported peaks at 22 years (Hale cycle), 33 years (three-cycle, TRC) and 79 years (Gleissberg, 1939). Rigozo et al. (2005) reported similar results. In an updated analysis, Kane (2006) reported peaks at 21, 27-39 and 94 years. (Kane, 2006, also pointed out that these peaks are not stationary. If the data are divided into three equal portions of $\sim 100$ years each, the peaks in the three samples differ considerably from each other, notably in the high periodicity region exceeding 11 years). However, the TRC invited attention only when Ahluwalia et al. (1996) and Ahluwalia (1998) noticed that in geomagnetic disturbance index $A_{p}$, the minimum values $A_{p}(\mathrm{~min})$ in cycle 17 and cycle 20 were very low, while the $A_{p}(\min )$ values for cycle 18 and 19 were higher than those for cycle 17 and the $A_{p}(\mathrm{~min})$ values for cycles 21 and 22 were higher than those for cycle 20. Thus, sequences of (low, high, higher; low high, higher) values were noticed, indicating a three-cycle periodicity. Ahluwalia (2003) had found a good correlation between $A_{p}$ and sunspot numbers 

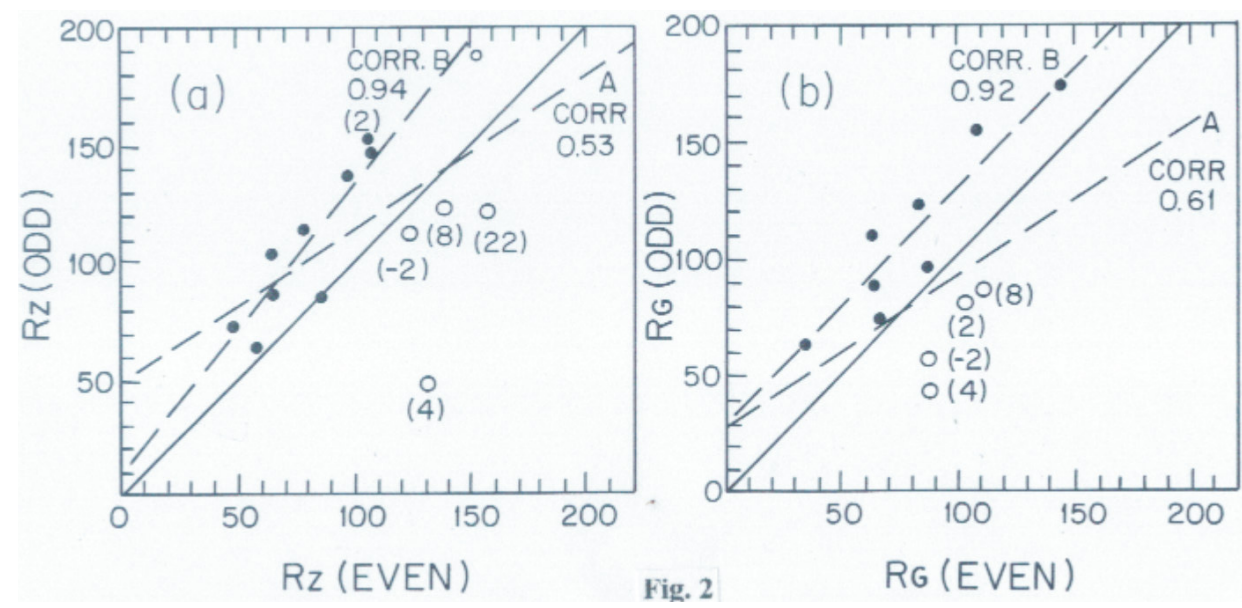

Fig. 2. (a) Plots of $R z(\max )$ of even cycles (abscissa) versus $R z(\max )$ of odd cycles (ordinate).The full line is a $45^{\circ}$ line, implying equal values for even and odd cycles. Points above the line (shown by dots) are when G-O rule proved correct. Points below (shown by circles) indicate G-O rule failures. The dashed regression line $\mathrm{A}$ is for all the points (dots and circles). The dashed regression line $\mathrm{B}$ is for all the dots only (i.e. excluding the circles). (b) Similar plots for $R_{G}(\max )$.

Table 3. The G-O effect for odd-even pairs. Successive odd(o)even(e) cycles (colums 1 and 3 ) and their $R z(\mathrm{o})$ and $R z(\mathrm{e})$ values (colums 2 and 4) are given and the percentage excess of the value of the odd cycle over the value of the preceding even cycle $[(R z(0)-$ $R z(\mathrm{e})) / R z(\mathrm{e})]$ is indicated (fifth column).

\begin{tabular}{ccccc}
\hline $\begin{array}{c}\text { Col.1 } \\
\text { Odd }\end{array}$ & 2 & 3 & 4 & $\begin{array}{c}5 \\
\text { Even } \\
\text { cycle }\end{array}$ \\
\hline cycle & $R z(\mathrm{o})$ & {$[(R z(\mathrm{e})-R z(\mathrm{o})] / R z(\mathrm{o})$} \\
\hline-3 & 63 & -2 & 122 & 93.7 \\
-1 & 111 & 0 & 83 & -25.2 \\
1 & 86 & 2 & 106 & 23.3 \\
3 & 154 & 4 & 132 & -14.3 \\
5 & 48 & 6 & 46 & -4.2 \\
7 & 71 & 8 & 138 & 94.4 \\
9 & 125 & 10 & 96 & -23.2 \\
11 & 139 & 12 & 64 & -54.0 \\
13 & 85 & 14 & 64 & -24.7 \\
15 & 104 & 16 & 78 & -25.0 \\
17 & 114 & 18 & 152 & 33.3 \\
19 & 190 & 20 & 106 & -44.2 \\
21 & 155 & 22 & 158 & 1.9 \\
& & & Mean & 2.4 \\
& & & Std. Error & 36.1 \\
\hline
\end{tabular}

and since the $A_{p}$ (min) for the beginning of cycle 23 was low, he predicted a low value of sunspot maximum for cycle 23 , which came true (value for cycle 23 was lower than that of cycle 22), in spite of strong criticism from some astronomers who had predicted a strong cycle 23 . The even-odd pair 2223 thus violated the G-O-K rule.
What happened to the G-O-K rule during cycles 17-23? Figure 3 shows the $R z(\max )$ values for successive cycles. $\left(R_{G}\right.$ values are not shown as the results were predominantly the same during the early part, cycles -4 to -1 ). The following may be noted in Fig. 3a:

1. The major fluctuations of $R z(\max )$ seem to have intervals of several cycles (Gleissberg cycles, more so in the earlier data), not much from cycle to cycle. Hence, the G-O-K rule (successive ups and downs in even-odd cycles) should be rather small. The positive G-O-K rule results are marked by thick lines. The negative rule results (failures) are marked by circled crosses.

2. The TRCs (two consecutive increases) are seen often, more so in recent cycles. These sequences are labeled as TRC, with the initial low value indicated by a big dot. These sequences include some positive G-O-K events.

The G-O-K rule is for even-odd pairs, not for odd-even pairs. Cycle 17 was odd and the G-O-K rule had no prediction for the next even cycle 18. But the TRC pattern (cycle 17 low, cycle 18 high, cycle 19 higher), fitted well with the G-O-K rule for the even-odd pair 18-19. In the pair 19-20, cycle 20 was low but the G-O-K rule had nothing to reveal, as it was an odd-even pair. But the TRC pattern (cycle 20 low, cycle 21 high, cycle 22 higher) fitted well with the G-O-K rule for the even-odd pair 20-21. For the odd-even pair 21-22, the G-O-K rule had nothing to reveal, so cycle 22 could be anything. The problem came when the next TRC pattern prediction would be (cycle 23 low, cycle 24 high, cycle 25 higher). Cycle 23 turned out to be low, thus adhering to the TRC pattern, but this violated the G-O-K rule for the even-odd pair 22-23. Thus, the TRC and G-O-K rules are not invariably contradictory. They would be contradictory once in a while, 


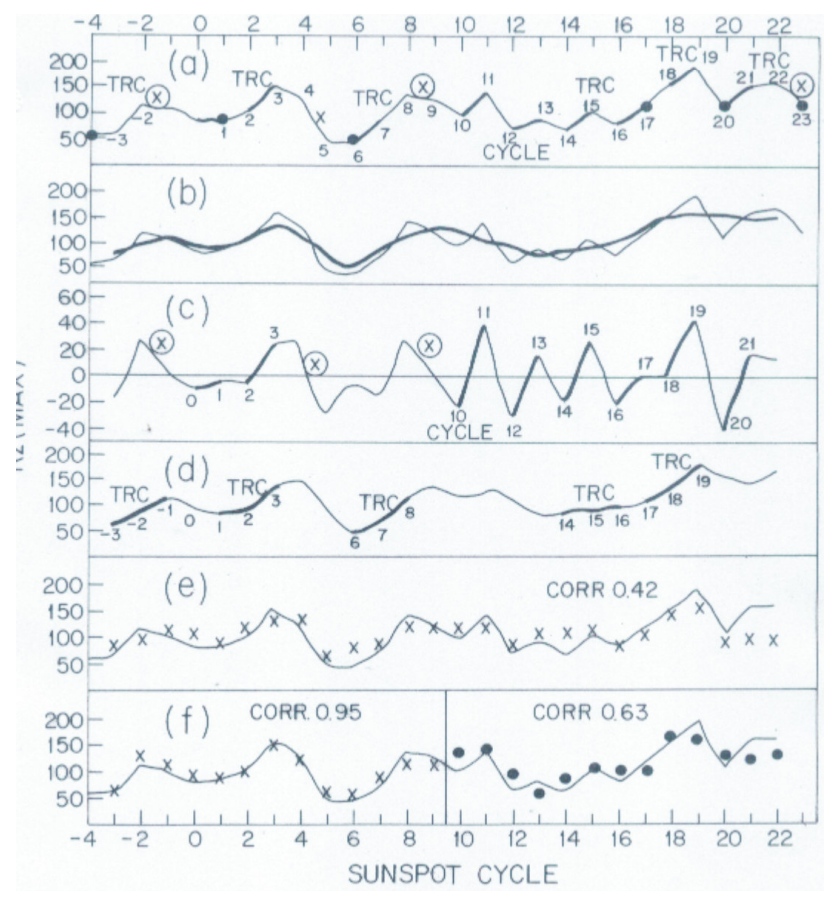

Fig. 3. (a) Plot of $R z$ (max) for cycles -4 to 23 . The even-odd pairs which obeyed the G-O rule are marked by thick lines and those disobeying the G-O rule are marked by circled crosses. The 3-cycle sequences TRC are labeled, their starting points indicated by big dots, (b) Plot of $R z$ (max) series (thin line) and the running mean over three consecutive cycles (long-term trend, superposed thick line), (c) Residues when the 3-cycle running mean is subtracted from the $R z(\max )$ series. Thick lines and crosses indicate evenodd pairs obeying and disobeying G-O rule, (d) 2-cycle running means, bringing out the TRC sequences prominently, (e) Original $R z(\max )$ series (full line) and the series reconstructed from spectral components (crosses), (f) original series and the reconstructed series, separately for the first half (crosses) and the second half (big dots).

probably after every 6-7 cycles, and then, the G-O-K rule failed and the TRC pattern prevailed.

Figure $3 \mathrm{~b}$ shows the $R z(\max )$ series as in Fig. 2a but with a superimposed plot of three-cycle averages (thick line), roughly representing a long-term trend. If this trend is subtracted from the original $R z(\max )$ series, the residues should bring out significant short-term cycle-tocycle changes. These residues are shown in Fig. 3c (data for cycles 22 and 23 are lost). The positive G-O-K rule results are shown by thick lines and the negative G-O-K rule results (failures) are shown by circled crosses. In the latter half of the data, there is a good sequence of Hale cycles 11-21 (alternate high and low cycles), but the magnitudes are small (note the expanded ordinate scale). As mentioned earlier, these increases vary in a large range (short and long thick lines) and predictions would have large uncertainties.
Table 4. $R z(\max )$ values for 3 -cycle sequences.

\begin{tabular}{ccccccc}
\hline & \multicolumn{3}{c}{$R z(\max )$} & \multicolumn{3}{c}{ Differences } \\
Cycles & 1 & 2 & 3 & 1 & 2 & 3 \\
\hline $2,3,4$ & 106 & 154 & 132 & 0 & 48 & 26 \\
5.6 .7 & 48 & 46 & 71 & 0 & -2 & 23 \\
$8,9,10$ & 138 & 126 & 96 & 0 & -12 & -42 \\
$11,12,13$ & 139 & 64 & 85 & 0 & -75 & -54 \\
$14,15,16$ & 64 & 104 & 78 & 0 & 40 & 14 \\
$17,18,19$ & 114 & 152 & 190 & 0 & 38 & 76 \\
$20,21,22$ & 106 & 155 & 158 & 0 & 49 & 52 \\
Mean & 102 & 114 & 116 & 0 & 12 & 14 \\
Std. Dev. & 26 & 37 & 38 & 0 & 36 & 35 \\
\hline
\end{tabular}

Figure $3 \mathrm{~d}$ shows the running average over two cycles so that the G-O-K effects are nearly eliminated. Some TRCs are now clearly seen, some isolated and some continuous. Their amplitudes are larger than the amplitudes of the Hale cycles in Fig. 3c and hence in Fig. 3a, the Hale cycles look as tiny waves embedded in TRCs.

\section{Prediction for cycle 24 based on TRC}

The TRC waves were strong for cycles 17-23 but weak for earlier periods. An average would be a good guide as to what to expect in the future. Table 4 shows the $R z$ (max) for 3 -cycle sequences starting at cycle 2 , and the differences between the starting cycle and the next two cycles. Their means and standard deviations are given at the bottom. As can be seen, the second and third cycles have values 12 and 14 above the starting cycle, but the standard errors are very large, $\sim 35$. Since cycle 23 was 120 , cycle 24 would be $132 \pm 36$ and cycle 25 would be $134 \pm 35$. These estimates are higher than those obtained by the G-O-K rule, but the errors are so large that anything between 60 and 170 is possible, not at all a meaningful prediction.

\section{Multiple periodicities: spectral analysis}

If more than one periodicity exists, a visual inspection can give misleading indications. A better way is to undertake a spectral analysis. Using MEM (Maximum Entropy Method, Burg, 1967; Ulrych and Bishop, 1975) in conjunction with MRA (Multiple Regression Analysis MRA, Bevington, 1969) as outlined in Kane and Trivedi (1982), the series in Table 1 were subjected to spectral analysis. The results are given in Table 5. For the whole series (28 data points, cycles -4 to 23 ), the reconstruction (crosses) is shown in Fig. $3 \mathrm{e}$ along with the original series (full lines). In the early part, the matching is good, but for the later portion (cycles 13 onwards), the fit is not good. The periodicities observed were 
Table 5. Periodicities (in solar cycles) and their amplitudes (units of sunspot number) with standard errors, for the whole series of $R z$ (max) (cycles -4 to 22), and for the first half (cycles -4 to 9) and second half (cycles 10-22).

\begin{tabular}{ccccccccc}
\hline \multicolumn{3}{c}{ Whole series, correlation } & 0.42 & \multicolumn{3}{c}{ First half, correlation 0.95 } & \multicolumn{3}{c}{ Second half, correlation 0.63 } \\
$\begin{array}{c}\text { Periodicity } \\
\text { (cycles) }\end{array}$ & $\begin{array}{c}\text { Amplitude } \\
\text { (sunspot No.) }\end{array}$ & $\begin{array}{c}\text { Std. } \\
\text { Error }\end{array}$ & $\begin{array}{c}\text { Periodicity } \\
\text { (cycles) }\end{array}$ & $\begin{array}{c}\text { Amplitude } \\
\text { (sunspot No.) }\end{array}$ & $\begin{array}{c}\text { Std. } \\
\text { Error }\end{array}$ & $\begin{array}{c}\text { Periodicity } \\
\text { (cycles) }\end{array}$ & $\begin{array}{c}\text { Amplitude } \\
\text { (sunspot No.) }\end{array}$ & $\begin{array}{c}\text { Std. } \\
\text { Error }\end{array}$ \\
\hline 2.17 & 13.2 & 10.5 & & & & & & \\
2.49 & 8.4 & 10.5 & 2.71 & 9.2 & 4.3 & & & \\
3.75 & 13.8 & 10.1 & & & & 3.72 & 25.3 & 11.7 \\
5.14 & 19.7 & 10.2 & 4.88 & 40.7 & 4.3 & & & \\
7.96 & 18.9 & 10.4 & & & & & & \\
& & & 9.91 & 19.2 & 4.4 & 10.38 & 37.0 & 12.2 \\
\hline
\end{tabular}

2.17 cycles (almost the Hale cycle of 22 years), 2.49 cycles ( $\sim 28$ years), 3.75 cycles ( $\sim 41$ years), 5.14 cycles ( $\sim 55$ years) and 7.96 cycles ( $\sim 87$ years, probably the Gleissberg cycle). (Note that there is no 33-year periodicity). However, the standard errors are large and the correlation between the original and the reconstructed series is only 0.42 . So, there could be considerable errors in these numbers.

In this methodology, there is a basic assumption that the periodicities are stationary throughout the series. To check whether this is really so, the spectral analysis was done separately for the first half (14 data points, cycles -4 to 9 ) and second half (13 data points, cycles 9 to 22 , cycle 23 left out to check prediction). The results are given in Table 5. Also, in Fig. 3f, the original series (full line) and the reconstructed series are shown (crosses for the first half and dots for the second half). As can be seen, in the first half, the matching is very good, with a correlation of 0.95 . There is no periodicity corresponding to the Hale cycle ( 2.0 cycles, $\sim, 22$ years). There is a periodicity of 2.71 cycles ( $\sim 30$ years), which is almost 3 cycles but not quite, and is barely significant. But a very significant periodicity is at 4.88 cycles ( $\sim 54$ years). (This is seen as two waves of $\sim 5$ cycles each in Fig. $3 \mathrm{f} \mathrm{left}$ half). There is also a periodicity of 9.9 cycles $(\sim 110$ years, earlier reported by Komitov and Kaftan, 2003 also). Thus, a clear, unique Gleissberg cycle of $\sim 80$ years is not seen. In the second half, the matching is reasonably good but the end points (cycles 21,22 ) have a mismatch. The correlation is only 0.63 , so prediction for cycle 23 by extrapolating periodicities would not be justified and hence, was not attempted. There are only two significant periodicities, 3.72 cycles $(\sim 40$ years) and 10.38 cycles ( $\sim 110$ years). Thus again, a clear, unique Gleissberg cycle of $\sim 80$ years as mentioned in Gleissberg (1939) is not seen. While this appeared reasonable at the time of that publication, the cycles since then have indicated longer period fluctuations for the more recent cycles (Hathaway and Wilson, 2004). How reliable these results are, is anybody's guess, but they certainly show that the sunspot series is not homogeneous and hence, results obtained from one interval may not be valid for another interval. In particular, the Hale cycle effect is almost negligible and the value of the results, based on odd-even differences, should be considered unreliable.

\section{Long time-scale variations}

In the results presented so far, the correlations have been low, in the range $0.3-0.6$, mostly insignificant, and this resulted in large standard errors in the regression coefficients and more so in the predictions, rendering the predictions unreliable. The general problem for all the predictions based on the observed data series (solar, geomagnetic etc.), comes from the fact that they do not take into account the long timescale variations of solar activity and the corresponding supercentennial and super-millennial oscillations like the 200-210 year and 2200-2400 year modulations. The latter are very well detectable in historical records (Schove's series and "cosmogenic" isotope data sets). In this regard, it may be pointed out that the current epoch is located at a very special timing in terms of long time-scale dynamics in solar activity. It is at the initial increasing phase of the 2200-2400 year (Halstadtzeit) solar cycle. Moreover, the period AD 19402000 is very peculiar, not only in the present Halstadtzeit cycle, but also for the last $\sim 8000$ years as was pointed out by Solanki et al. (2004). The solar activity for this epoch was extremely high compared to the typical range of variability. On this basis, all predictions based on the observed data for the last $70-80$ to $\sim 300$ years, are affected by this situation. They depend very much on the types of data used and the methods of analysis and the prediction varies from a very low cycle $24(R z(\max ) 50-60)$ (Badalyan, 2000; Obridko and Sykora, 2005; Archibald, 2006, etc.) to a very powerful one $(R z(\max ) \sim 150)$ (Dikpati et al., 2006; Du, 2006, etc.). The scenarios based on the observed data are in serious contradiction. On the contrary, predictions based on indirect "historical" data are in agreement for the forthcoming supercentennial solar minimum during the 21 st century (Komitov and Bonev, 2001; Komitov and Kaftan, 2003, 2004; Solanki et al., 2004; Bonev et al., 2004; Ogurtsov, 2005) and it is 
independent from what types of historical data $(14 \mathrm{C}, 10 \mathrm{Be}$ or Schove series data) or methods have been used. Komitov and Kaftan's studies are based on multiple periodicity. In this study, as well as in the other ones (Komitov and Bonev, 2001), it has been pointed out that Bonev, Penev and Selo (2004) and Komitov (2007) found that there is an amplitude modulation in shorter solar cycles coming from longer ones. As a result, many of the short time scale solar activity oscillations could have been affected in the last $\sim 300-350$ years. Therefore, a simple extrapolation, especially of short cycles (less than $\sim 100$ years) during the present transient epoch of the $2200-2400$ year cycle for solar activity prediction, is a risky procedure. On the other hand, the risk is not so large when longer cycles (bi-centennial) or more duration are used.

During the present epoch ( AD 2000-2050), a transition from the initial active to the "quiet" phase ("plateau") of the 2200-2400 year cycle should occur. For cycles 19-23, the $R z(\max )$ were $\sim 201,111,165,159,122$. A downtrend is not exactly obvious, but for the next few cycles, the values are expected to be less than the value 122 for cycle 23 . Some workers have expressed the following opinions: Schatten and Tobiska (2003) claim that solar activity will decrease after cycle 24 and will be heading for a Maunder Minimum in the next few decades. Duhau (2003) mentions that solar activity is in a declining episode, which started about 1993. A very similar prediction has been made more recently by Clilverd (2005) based on the modeling of low-frequency solar oscillations. He says that the peak sunspot prediction for cycle 24 will be significantly smaller than for cycle 23 , and peak sunspot numbers are predicted to be $\sim 45 \pm 27$. However, the model also predicts a recovery during the middle of the century to more typical solar activity cycles with peak sunspot numbers of $\sim 120$.

\section{Conclusions and discussion}

An examination of the $R z(\max )$ values of all cycles revealed fluctuations of various intervals in the high periodicity region (exceeding 11 years), namely 2 cycles (Hale 22 year), 3 cycles (TRC, three-cycle) and longer intervals. The following was noted:

1. Among these, the 2-cycle intervals had the smallest amplitudes. According to the G-O-K (Gnevyshev-Ohl and Kopecky) rule (Gnevyshev and Ohl, 1948; Kopecky, 1950), the even-numbered International Sunspot Number series 11-year cycles have been followed by higher amplitude odd-numbered ones. However, as pointed out earlier by Komitov and Bonev (2001), there were some failures of this rule. We found that for the data of 28 cycles, from cycle -4 (maximum in 1705) to cycle 23 (maximum in 2000), from the fourteen even-odd pairs $(-4,-3),(-2,-1),(0,1),(2,3)$ to $(22,23)$, four pairs showed failure of the G-O-K rule (odd cycles were weaker than the preceding even cycles). Thus, the failure rate was $\sim 29 \%$. In the remaining ten pairs, the magnitudes of the odd cycles were well-correlated with the magnitudes of the preceding even cycles, and a good prediction potential existed; provided one could tell (impossible) whether it would be a normal pair obeying the G-O-K rule or a possible failure case, notably like the recent odd cycle 23 which had a magnitude of only 120 , much smaller than the magnitude of the preceding even cycle 22 which had a magnitude of 158 . (The low value of cycle 23 has proved to be a concern for many prediction schemes; see the review by Kane, 2001b). Nevertheless, using the regression equations for the G$\mathrm{O}-\mathrm{K}$ rule, the known value for cycle 23 could be utlised for predictions for cycle 24 . The estimate was $106 \pm 42$ and $112 \pm 36$ (by a slightly different approach). Since the correlations are poor, the errors are large, rendering the predictions not very meaningful.

2. The three-cycle sequence was much stronger (TRC, low, high, higher), and the 2-cycle oscillations were embedded into the TRC until the G-O-K rule failures, like cycle 23 , occurred. The possibility of a three-cycle periodicity had been indicated earlier in spectral analyses (Kane, 1977, 2006; Rigozo et al., 2005); but it was Ahluwalia et al. (1996) and Ahluwalia (1998) who noticed it in the geomagnetic disturbance index $A_{p}$ and in the suspot maxima. The values $A_{p}(\mathrm{~min})$ in cycle 17 and cycle 20 were very low, while the $A_{p}(\mathrm{~min})$ values for cycles 18 and 19 were higher than those for cycle 17 and the $A_{p}(\mathrm{~min})$ values for cycles 21 and 22 were higher than those for cycle 20 . This was seen in the sunspot numbers also, and since the $A_{p}(\mathrm{~min})$ for the beginning of cycle 23 was low, Ahluwalia et al. (1996) and Ahluwalia (1998) predicted a low value of sunspot maximum for cycle 23, which came true (value for cycle 23 was lower than that of cycle 22). Ahluwalia et al. (1996) and Ahluwalia (1998) were very fortunate, firstly, by detecting the 17 (low), 18 (high), 19 (higher); 20 (low), 21 (high), 22 (higher) patterns, secondly, by predicting that cycle 23 would be low, and thirdly, by getting this confirmed by observation. When we examined the earlier data, the immediately preceding sequence was rather uncertain, namely, 14 (low), 15 (high), but 16 (not higher). Before that for seven cycles (cycles 814), there were no TRC sequences at all. Also, still further back, during the twelve cycles -4 to 7 , there were only three isolated sequences, namely, cycle 6 (low), 7 (high), 8 (higher), cycle 1 (low), 2 (high), 3 (higher), and cycle -4 (low), -3 (high), -2 (higher) (last one not reliable). Using the observed value 120 for cycle 23 as a start of a new TRC sequence, a prediction could be made for the next two cycles. The prediction for cycle 24 was $132 \pm 36$ and for cycle 25 , it was $134 \pm 35$. Again, the correlations were small, errors are large, and 
the prediction cannot be considered satisfactory. However, the more than two dozen predictions made for cycle 24 by several methods (see the list in Kane, 2007b) have similar large errors. Thus, all these predictions are equally unsatisfactory. Incidentally, if the physical basis is ignored and only statistics are considered, the average for the 23 cycles works out to $114 \pm 41$, again not very different from all other predictions, with a large error. Thus, our present-day prediction capacity seems to be similar to an assumption of randomness, and this random prediction $114 \pm 41$ for all cycles will come out true at least in $66 \%$ of cases ( \pm one $\sigma$ level).

3. An inspection of the data plots showed that the patterns changed from one interval to another. Spectral analysis showed that in the first half (cycles -4 to 9), larger periodicies (reminiscent of the Gleissberg cycle of $\sim 80$ years) prevailed. The original series and the series reconstructed from the detected spectral periodicities matched very well (correlation 0.95 ), but in the latter half, periodicities were different and the matching was not so good (correlation 0.63). In particular, the points for the recent cycles 21,22 seemed to deviate considerably from the reconstructed series, thus introducing unreliability in predictions for future by using the method of extrapolation of periodicities.

The prediction of the strengths of future sunspot cycles has received considerable attention in recent decades. For cycle 23, Obridko et al. (1994) compiled the forecasts and divided these into two main groups: (i) those based on internal regularities in a pair of cycles (e.g. the 22-year cyclicity), and (ii) those also using the secular cycle and the sunspot number variations for many years. They observed that group (i) had high values and group (ii) had moderate and low values. Soon after, NOAA Space Environment Center (SEC) recruited a scientific panel to assess the likely development of cycle 23 and their report entitled "Solar cycle 23 Project: Summary and Panel Findings", later published as Joselyn et al. (1997), mentioned (i) a range of 160-200 of $R z(\max )$ of cycle 23 as obtained by considering the even/odd behaviour, and (ii) a range $110-160$ of $R z(\max )$ by other methods. The observed value of 120 brought discredit to many prediction schemes in (i), some of which claimed to have a very sound physical basis. For cycle 24 also, many predictions have been made, again in a very wide range (see list in Kane, 2007b). A Solar Cycle 24 Prediction Panel, composed of international scientists and presided by Douglas Biesecker (http://www. sec.noaa.gov/SolarCycle/SC24/index.html) issued a consensus opinion on 25 April 2007, that the cycle would commence in March 2008 ( \pm 6 months) and two consensus opinions, that the solar maximum would be $140 \pm 20$ in October 2011 or $90 \pm 10$ in August 2012. However, this does not resolve the problem of ambiguity. Whatever value is finally observed in 2010-2011, it will bring discredit to some methodology. It seems that while we have learnt a lot about Solar
Physics in recent years, the problem of guessing the nature of the variations of solar activity is still not reliable for studies and predictions based on relatively short instrumental data series, while the situation with solar "historical" data sets is much better.

Acknowledgements. This work was partially supported by FNDCT, Brazil under contract FINEP-537/CT.

Topical Editor R. Forsyth thanks V. N. Obridko, J. Javaraiah, and another anonymous referee for their help in evaluating this paper.

\section{References}

Ahluwalia, H. S.: The Predicted Size of Cycle 23 Based on the Inferred Three Cycle Quasiperiodicity of the Planetary Index $A_{p}$ J. Geophys. Res., 103, 12 103-12 109, 1998.

Ahluwalia. H. S.: Meandering path to solar activity forecast for cycle 23, in: Solar Wind Ten: Proc. Tenth International Solar Wind Conf., edited by: Velli, M., Bruno, R., and Malara, F., pp. 176179, published by American Institute of Physics, 2003.

Ahluwalia, H. S., Xue, S. S., Fikani, M. M., and Gazis, P. R.: Long term variability of the solar wind speed, Solar Wind Eight Conf. Paper, AIP Conf. Proc., edited by: Winterhalter, D., Gosling, J, T., Kurth, W. S., and Neugebauer, M., Woodbury, 382, 469 p., 1996.

Archibald, D.: Solar Cycles 24 and 25 and Predicted Climate Response, Energy and Environment, 17, 29-35, 2006.

Badalyan, O. G., Obridko, V. N., and Sykora, J.: Brightness of the coronal green line and prediction for activity cycles 23 and 24 Solar Phys., 199, 421-435, 2000.

Bevington, P. R.: Data Reduction and Error Analysis for the Pbysical Sciences., McGraw-Hill, New York, pp. 164-176, 1969.

Bonev, B., Penev, K., and Sello, S.: Long-term solar variability and the solar cycle in the XXI century, Astrophys. J. Lett., 605, 8184, 2004

Burg, J. P.: Maximum Entropy Spectral Analysis, paper presented at the 37th Meeting, Society of Exploration Geophysics, Oklahoma City, October, 1967.

Clilverd, M.: Prediction of solar activity the next 100 years, Solar Activity: Exploration, Understanding and Prediction, Workshop in Lund, Sweden, 2005.

Dikpati, M., de Toma, G., and Gilman, P. A.: Predicting the strength of solar cycle 24 using a flux-transport dynamo-based tool, Geophys. Res. Lett., 33, L05102, doi:10.1029/2005GL025221, 2006.

Du, Z. L.: Relationship Between Solar Maximum Amplitude and Max-Max Cycle Length, Astronom. J., 132, 1485-1489, 2006.

Duhau, S.: An early prediction of maximum sunspot numbers in solar cycle 24, Solar Phys., 213, 203-212, 2003.

Gleissberg, W.: A long-periodic fluctuation of the sun-spot numbers, The Observatory, 62, 158-159, 1939.

Gnevyshev, M. N. and Ohl, A. I.: On the 22-year cycle of solar activity, Astronom. J., 38, 15-20, 1948.

Hathaway, D. H. and Wilson, R. M.: What the sunspot record tells us about space climate?, Solar Phys., 224, 5-19, 2004.

Hoyt, D. V. and Schatten, K. H. (Eds.): Observations of the sun, in: The role of the sun in climate change, New York: Oxford University Press, Oxford, 279 pp., 1997. 
Hoyt, D. V. and Schatten, K. H.: Group sunspot numbers: a new solar activity reconstruction, Solar Phys., 179, 189-219, 1998a.

Hoyt, D. V. and Schatten, K. H.: Group sunspot numbers: a new solar activity reconstruction, Solar Phys., 181, 491-512, 1998b.

Javaraiah, J.: Long-term variations in the solar differential rotation, Solar Phys., 212, 23-49, 2003.

Javaraiah., J.: Sun's retrograde motion and violation of even-odd cycle rule in sunspot activity, Monthly Notices of the Royal Astronomical Society, 362, 1311-1318, 2005.

Javaraiah, J.: North-south asymmetry in solar activity: predicting the amplitude of the next solar cycle, Monthly Notices of the Royal Astronomical Society: Letters, 377, L34-L38, 2007.

Javaraiah, J., Bertello, L., and Ulrich, R. K.: An interpretation of the differences in the solar differential rotation during even and odd sunspot cycles, Astrophys. J., 626, 579-584, 2005.

Joselyn, J. A., Anderson, J. B., Coffey, H., Harvey, K., Hathaway, D., Heckman, G., Hildner, E., Mende, W., Schatten, K., Thompson, R., Thomson, A. W. P., and White, O. R.: Solar Cycle 23, Panel Achieves Consensus Prediction of Solar Cycle 23, Eos Transactions AGU, 78, 205, 1997.

Kane, R. P.: Power spectrum analysis of solar and geophysical parameters, J. Geomagn. Geoelectr., 29, 471-495, 1977.

Kane, R. P.: Examination of the three-cycle quasiperiodicity of geomagnetic indices in relation to prediction of the size of cycle 23 , J. Geophys. Res., 106, 25 125-25 131, 2001a.

Kane, R. P.: Did predictions of the maximum sunspot number for solar cycle 23 come true?, Solar Phys., 202, 395-406, 2001 b.

Kane, R. P.: Some implications using the group sunspot number reconstruction, Solar Phys., 205, 383-401, 2002.

Kane, R. P.: Some methods of spectral analysis: Comparison with MEM-MRA combination, Appl. Math. Comput., 181, 949-957, 2006.

Kane, R. P.: Solar cycle predictions based on solar activity at different solar latitudes, Solar Phys., 246, 471-485, 2007a.

Kane, R. P.: A Preliminary Estimate of the Size of the Coming Solar Cycle 24, based on Ohl's Precursor Method, Solar Phys., 243, 205-217, 2007b.

Kane, R. P. and Trivedi, N. B.: Relationship between sunspot numbers during years of sunspot maximum and sunspot minimum, Solar Phys., 68, 135-139, 1980.

Kane, R. P. and Trivedi, N. B.: Comparison of Maximum Entropy Spectral Analysis (MESA) and Least-Squares Linear Prediction (LSLP) methods for some artificial samples, Geophysics, 47, 1731-1736, 1982.

Komitov, B.: The Schove's series. Centural and Supercentural variations of the solar activity. Relationships between adjacent 11year cycles, Bulgarian Geophys. J., 23, 74-82, 1997.

Komitov, B.: The supercenturial solar minima and their preceding phenomena, Astronomical Invest, 9, 112-120, 2007.

Komitov, B. and Bonev, B.: Amplitude Variations of the 11-year Solar Cycle and the Current Maximum 23, Astrophys. J. Lett., 554, L119-L122, 2001.
Komitov, B. and Kaftan, V.: Solar Activity Variations for the Last Millennia. Will the Next Long-Period Solar Minimum be Formed?, Int. J. Geomagn. Aeron., 43, 553-561, 2003.

Komitov, B. P. and Kaftan, V. I.: The Sunspot Activity in the Last Two Millennia on the Base of Indirect and Instrumental Indexes. Time Series Models and Their Extrapolations for the 21st Century, in: Proceedings IAUS 223 "Multi-Wavelength Investigations of the Solar Activity:, edited by: Stepanov, A. V., Benevolenskaya, E. E., and Kosovichev, A. G., Cambridge University Press, pp. 115-116, 2004.

Kopecky, M.: Cycle de 22 ans de l'activité solaire, B. Astron. Institute of Czechoslovakia, 2, 14-16, 1950.

McKinnon, J. A.: Sunspot numbers 1610-1985, based on "The sunspot activity in the years 1610-1960", revised by J. A. Mckinnon, UAG Report 95, NOAA, Boulder, Colorado, USA, pp 112, 1987.

Obridko, V. N.: Some comments on the problem of solar cycle prediction, Solar Phys., 156, 179-190, 1995.

Obridko, V. N., Oraevsky, V. N., and Allen, J. H.: International Decade of Solar Sycle Studies (IDSCS), in: Solar-terrestrial energy program. The initial results from STEP Facilities and Theory Campaigns, edited by: Baker, D. N., Papitashvili, V. O., and Teague, M. J., COSPAR Colloquia Series, vol. 5, pp. 557-573, Pergamon Press, Oxford, 1994.

Ogurtsov, M.: On the Possibility of Forecasting the Sun's Activity Using Radiocarbon Solar Proxy, Solar Phys., 231, 167-176, 2005.

Rigozo, N. R., Echer, E., Nordemann, D. J. R., Vieira, L. E. A., and de Faria, H.: Comparative study between four classical spectral analysis methods, Appl. Math. Comput., 168, 411-430, 2005.

Schatten, K. H. and Tobiska, W. K.: Solar Activity Heading for a Maunder Minimum?, B. Am. Astron. Soc., 35, 817, 2003.

Schov, D. J.: The sunspot cycle 649 BC to 2000 AD, J. Geophys. Res., 60, 127-146, 1955.

Schov, D. J.: Sunspot cycles, Stroudsnurg: Hutchinson Ross, 1983.

Solanki, S. K., Usoskin, I. G., Kromer, B , Schussler, M., and Beer, J.: Unusual activity of the Sun during recent decades compared to the previous 11000 years, Nature, 431, 1084-1087, 2004.

Ulrych, T. J. and Bishop, T. N.: Maximum entropy spectral analysis and utoregressive decomposition, Rev. Geophys., 13, 183-200, 1975.

Waldmeier, M.: The sunspot-activity in the years 1610-1960, Schulthess \& Co. AG, Zurich, 1961.

Wilson, R. M.: Bimodality and the Hale cycle, Solar Phys., 117, 269-278, 1988.

Wilson, R. M. and Hathaway, D. H.: Comment on "The predicted size of cycle 23 based on the inferred three-cycle quasiperiodicity of the planetary index Ap" by H. S. Ahluwalia, J. Geophys. Res., $104,2555-2558,1999$. 